\title{
Fabrication and Characteristics of Blue-Green and Green LEDs using ZnSSe:Te Active Layers
}

\author{
Hong-Chan Lee L $^{\dagger}$ \\ (Received November 16, 2010; Revised November 26, 2010; Accepted November 26, 2010)
}

\begin{abstract}
Blue-green and green LEDs have been successfully fabricated grown by MBE, which has introduced the $\mathrm{ZnS}_{\mathrm{y}} \mathrm{Se}_{1-\mathrm{x}-\mathrm{y}}: \mathrm{Te}_{\mathrm{x}}(\mathrm{x}=0.04, \mathrm{y} \sim 0.11-0.14)$ ternary epilayer as an active layer. From the $I-V$ characteristics, the built-in voltage $(\sim 2.1 \mathrm{~V})$ is very small compared to other wide bandgap LEDs, such as commercial InGaN-based LEDs $(>3.2$ V). From the $C-V$ profiling, the effective carrier concentration in the p-type ZnMgSSe cladding layer was evaluated as $\sim 2.8 \times 10^{16} \mathrm{~cm}^{-3}$ for the present LEDs.
\end{abstract}

Key words: LED, Blue-Green, Green, ZnSSe:Te Active Layer, MBE

\section{Introduction}

Blue and green high-brightness LEDs (light emitting diodes) are highly desirable for numerous practical applications such as multicolor LED indicators, traffic control signals, full-color outdoor displays, general lighting, and TV-use edge-type LED backlight unit (BLU). Wide band gap II-VI semiconductor compounds have been regarded as very promising materials for the fabrication of both LEDs and laser diodes. Enormous progress have been achieved in the development of ZnSe-based II-VI blue- green lighter emitters in recent years [1, 2]. D.B Eason et al. reported world-class demonstrations of blue-green LEDs on II-VI heterostructure grown on ZnSe substrates $[1,2]$.
In other previous studies [3-5], the binary $\mathrm{ZnSe}_{1-\mathrm{x}}$ : $\mathrm{Te}_{\mathrm{x}}$ system seemed to be a good candidate material for efficient green light emitters. The binary system, however, has a large technical problem in terms of the control of both Te doping amount and defect densities. This problem is simply attributed to the lattice mismatch to GaAs substrates. We have overcome this problem by using a tenary $\mathrm{ZnS}_{\mathrm{y}} \mathrm{Se}_{1-\mathrm{x}-\mathrm{y}}: \mathrm{Te}_{\mathrm{x}}$ system where we have obtained highly improved crystal quality [6]. In this article, the fabrication process of blue-green and green light emitting diodes using the tenary $\mathrm{ZnS}_{\mathrm{y}} \mathrm{Se}_{1-\mathrm{x}-\mathrm{y}}$ : $\mathrm{Te}_{\mathrm{x}}$ system and their characteristics will be presented.

\footnotetext{
† Corresponding Author (Faculty of Energy \& Resources Engineering, Jungwon University, E-mail: leehc@jwu.ac.kr, Tel: 043)830-8623)
} 


\section{Experimental Procedure}

\subsection{Fabrication of LEDs}

ZnSSe:Te/ZnMgSSe double hetero(DH) structure LEDs were grown on $\mathrm{Si}$-doped GaAs (100) substrates by molecular beam epitaxy (MBE). The substrates were chemically etched before the growth. The surface oxide layer has been removed by heating up to $\sim 580^{\circ} \mathrm{C}$ in the $\mathrm{MBE}$ chamber before the growth. The growth temperature was $230^{\circ} \mathrm{C}$. The source materials were $\mathrm{Zn}(6 \mathrm{~N})$, Se $(6 \mathrm{~N}), \mathrm{ZnS}$ $(6 \mathrm{~N}), \mathrm{Mg}(6 \mathrm{~N}), \mathrm{ZnSe}(5 \mathrm{~N}), \mathrm{ZnTe}(6 \mathrm{~N})$, and $\mathrm{Te}(6 \mathrm{~N}) . \mathrm{ZnCl}_{2}$ (6N) was used as an n-type doping source, and the $\mathrm{p}$-type layers were obtained using active nitrogen $(5 \mathrm{~N})$ produced by a radio-frequency $(\mathrm{RF})$ plasma source, where $\mathrm{RF}$ power and N2 flow rate were $300 \mathrm{~W}$ and $0.2 \mathrm{sccm}$, respectively. The Te-doped ZnSSe ternary epilayer has introduced as an active layer, which is lattice-matched to the GaAs substrate.

The structure of the LEDs was $\mathrm{p}-\mathrm{ZnTe}$ (cap layer) / p-type ZnSe-ZnTe superlattice electrode / p-ZnSe / p-ZnMgSSe (top cladding layer) / i-ZnSSe:Te (active region) / $\mathrm{n}$-ZnMgSSe (bottom cladding layer) / n-ZnSe (buffer layer) on $n+-$ GaAs substrate. The superlattice electrode (SLE) layer [7] was grown, by alternating $\mathrm{p}-Z n S e$ and $\mathrm{p}$-ZnTe, i.e. multi-quantum well (MQW) layers, and formed to obtain a good ohmic contact. Evaporated Au was used to form a metal contact to the top ZnTe surface of each LED. Ohmic contact to the n-type layer was made at back surface of the GaAs:Si substrate with In-metal or $\mathrm{Au}-\mathrm{Ge}$. The LEDs were mounted with the $\mathrm{n}$-side down on a sample holder (TO5).

\subsection{Measurement of LEDs}

The crystallinity of the LEDs was surveyed at room temperature by $\mathrm{x}$-ray diffraction (XRD) with MAC Science 2 -axis Diffractometer. $\mathrm{Cu}-\mathrm{Ka} \quad(\lambda=1.5405 \AA)$ radiation was employed for this measurement. To investigate the electrical and optical characteristics was carried out that the injection current-applied voltage $(I-V)$ characteristics, electroluminescence (EL), and capacitance-biased voltage $(C-V)$ measurement.

\section{Results and Discussion}

\subsection{Crystallographic characteristics}

The crystal qualities of the ZnSSe:Te/ZnMgSSe DH-structure LEDs are investigated by $\mathrm{X}$-ray diffraction (XRD) measurement. Figure 1 shows the typical XRD patterns of ZnSSe:Te-based DH-structure LEDs. The XRD patterns of the LEDs show only the (400) diffraction.

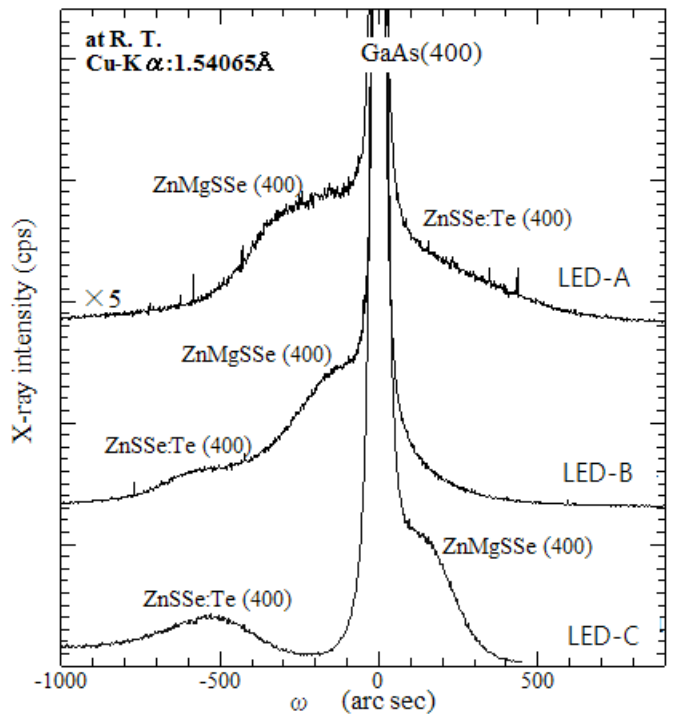

Figure 1: The typical XRD patterns of ZnSSe: Te-based DH-LEDs. Samples were measured at room temperature. 
As can be seen, the diffraction patterns of ZnSSe:Te active layers show good lattice-matched $(\Delta \mathrm{a} / \mathrm{a}\langle 0.41 \%)$ to GaAs substrate and also somewhat small values of full width at half maximum(FWHM) in XRD. The fact implies that the cladding layers have a good crystallinity. The diffraction patterns of ZnSSe:Te layer introduced as an active layer exhibit rather large values of FWHM and lattice-mismatch factor with respect to GaAs substrate.

Table 1: The list of FWHM(arcsec) and lattice mismatch factor $(\triangle \mathrm{a} / \mathrm{a})$ by means of $\mathrm{x}$-ray diffraction.

\begin{tabular}{|l|c|c|c|}
\hline & $\begin{array}{c}\text { FWHM } \\
\text { ZnSSe:Te }\end{array}$ & $\begin{array}{c}\text { FWHM } \\
\text { ZnMgSSe }\end{array}$ & $\begin{array}{c}\triangle \mathrm{a} / \mathrm{a}(\%) \\
\text { ZnSSe:Te }\end{array}$ \\
\hline LED-A & 350 & 230 & +0.41 \\
\hline LED-B & 340 & 310 & +0.32 \\
\hline LED-C & 410 & 330 & -0.15 \\
\hline
\end{tabular}

\subsection{EL and Electrical Properties of LEDs}

\subsubsection{Electroluminescence Characteristics}

Room temperature EL (electroluminescence) spectra for ZnSSe:Te-based DH-LEDs -A, $-B$, and $-C$, emitting in the blue-green and green color range, is shown in Figure 2. Data for the peak position and FWHM for each of the EL spectra are presented in the figure. The optical properties (temperature dependent PL spectra) of $\mathrm{ZnS}_{y} \mathrm{Se}_{1^{-x-y}}: \mathrm{Te}_{x}(x=0.04, y=0.11)$ epilayers have already been reported [6]. When the peak wavelength of $\mathrm{Te}_{n} \quad(n \geq 2)$ cluster emission (dominant peak) of $\mathrm{Te}$-doped ZnSSe epilayers became $500 \mathrm{~nm}$ in temperature dependent PL spectra at 300 $\mathrm{K}$. The FWHM of the Te $\mathrm{T}_{\mathrm{n}}$ cluster emission of PL measurements of ZnSSe:Te epilayers was about $65 \mathrm{~nm}$. For LED-A, the EL emission peak, blue-green color, is
$500 \mathrm{~nm}(2.48 \mathrm{eV})$ which coincides with the peak wavelength of $\mathrm{Te}_{\mathrm{n}}$ cluster emission (dominant peak observed at $2.48 \mathrm{eV}$ ) studied in temperature dependent $\mathrm{PL}$ spectra [6]. The value of the FWHM of the EL of the ZnSSe:Te-based LED-A (56 $\mathrm{nm}$ ) is somewhat different from that of the $\mathrm{Te}_{\mathrm{n}}$ cluster emission of $\mathrm{ZnS}_{y} \mathrm{Se}_{1^{-x}-y}: \mathrm{Te}_{x}$ $(\mathrm{x}=0.04, \mathrm{y}=0.11)$ epilayers $(65 \mathrm{~nm})$ of the PL measurements.

On the other hand, for LED-B and $-\mathrm{C}$, the peaks wavelength $(525 \mathrm{~nm})$ of the $\mathrm{EL}$ are slightly different from that $(530 \mathrm{~nm})$ of temperature dependent PL measurements. The values of the FWHM of the EL (50 and $56 \mathrm{~nm}$ ) are differ a little from those of the $\mathrm{Te}_{\mathrm{n}}$ cluster emission of $\mathrm{ZnS}_{y} \mathrm{Se}_{1-x^{-} y}: \mathrm{Te}_{x}$ $(x=0.04, y=0.14)$ epilayers $(60 \mathrm{~nm})$ of the PL. In view of the peaks wavelength and FWHMs of peak emission of EL, these blue-green and green emission can be assumed to result from recombination between the electrons injected into the conduction band and holes injected into the valence band of the ZnSSe:Te active layer.

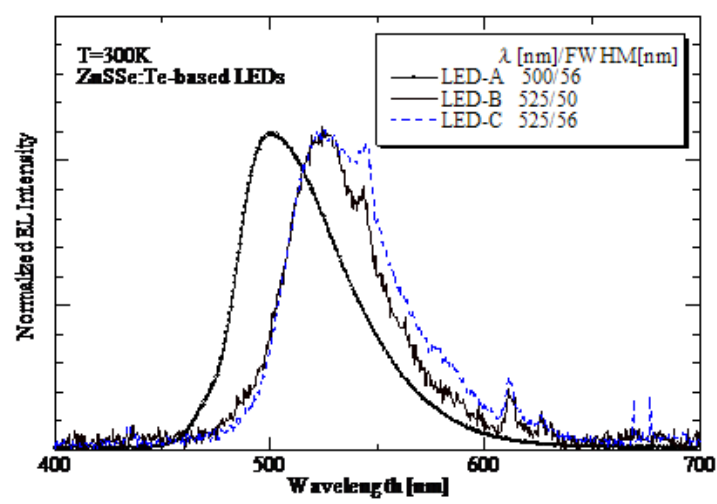

Figure 2: EL spectra for three ZnSSe:Te-based LEDs with blue-green and green light emission. The intensity of each spectrum is normalized to the respective peak intensity. 
Figure 3 shows the EL spectra of the ZnSSe:Te/ZnMgSSe DH-LED (LED-A) at forward currents of $2 \mathrm{~mA}, 10 \mathrm{~mA}$ and 30 $\mathrm{mA}$. The peak wavelength is $500 \mathrm{~nm}$ and the FWHM of peak emission is $56 \mathrm{~nm}$ at each current. The peak wavelength and the FWHM are almost constant under these DC-biased conditions. The fact is that $\mathrm{Te}_{\mathrm{n}}$ clusters play the role of recombination center in ZnSSe:Te films.

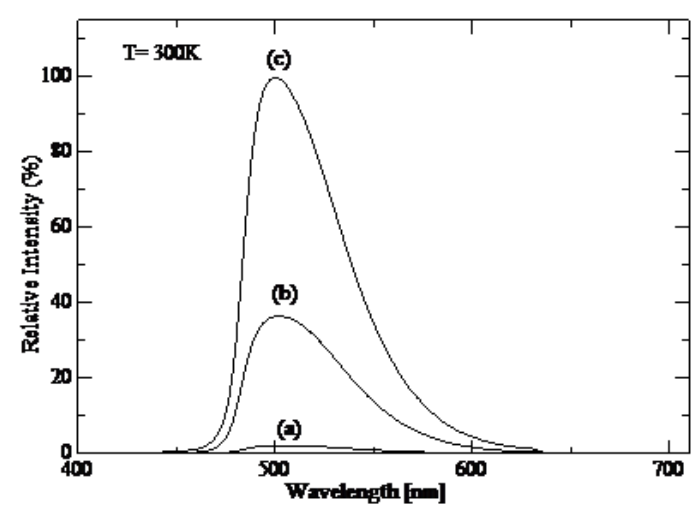

Figure 3: EL spectra of the $\mathrm{ZnSSe}: \mathrm{Te} / \mathrm{ZnMgSSe}$ double-heterostructure blue-green LED. Forward currents are (a) $2 \mathrm{~mA}$, (b) $10 \mathrm{~mA}$ and (c) $30 \mathrm{~mA}$ at $300 \mathrm{~K}$.

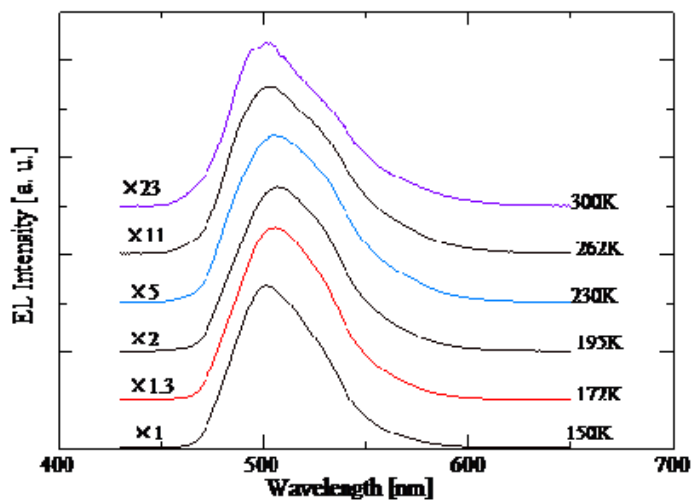

Figure 4: Temperature dependence of EL spectra of ZnSSe:Te-based blue-green LED.

The temperature dependence of EL spectra in the range from $150 \mathrm{~K}$ to the room temperature has been measured on the unaged ZnSSe:Te/ZnMgSSe DH-LED (LED-A), as shown in Figure 4. The variation tendency of the peak wavelength as a function of temperature for LED-A shows a similar tendency with that of the ZnSSe:Te epilayers described previously study [6].

\subsubsection{I-V characteristics and junction quality}

Typical dark current-voltage $(I-V)$ characteristics for LEDs $-\mathrm{A},-\mathrm{B}$, and $-\mathrm{C}$ are shown in Figure 5. For the case of LED-A, it is shown that the forward voltage is $2.1 \mathrm{~V}$ at $1 \mathrm{~mA}$. In case LED-B, however, the forward voltage $(2.5 \mathrm{~V})$ is larger in comparison with that of LED-A $(2.1 \mathrm{~V})$. This may be caused by the high resistivity of the superlattice electrode (SLE) layer. In general, the currentvoltage characteristics for a $\mathrm{p}-\mathrm{i}-\mathrm{n}$ diode can be written as Eq. (1):

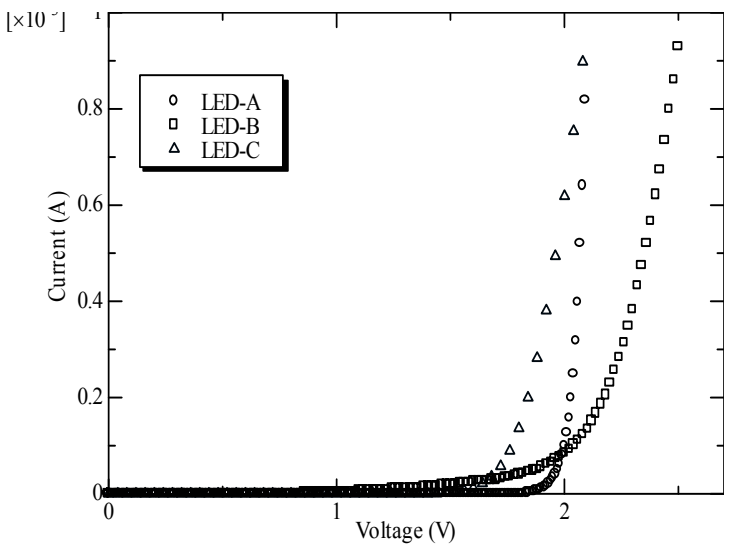

Figure 5: I-V characteristics of ZnSSe:Te-based LEDs.

$$
J=J_{s}[\exp (q V / n k T)-1]
$$

where

$$
n=\frac{q}{k T} \frac{1}{[\partial(\ln J) / \partial V]}
$$


Here $J_{s}$ is saturation current density at zero bias, $k$ is the Boltzmann constant, $T$ is the temperature in Kelvin, and $n$ is the ideality factor. From the $I-V$ characteristics, the ideality factors of 2.0 in the forward bias range of $1.5 \mathrm{~V}$ to $2.0 \mathrm{~V}$ were extracted for LED-A and LED-C. The built-in voltage $(\sim 2.1 \mathrm{~V})$, which is attained by the insertion of a new superlattice electrode ( $p$-ZnSe) $p$-ZnTe), is very small compared to other wide bandgap LEDs, such as commercial InGaN-based LEDs (>3.2 V) [8]. In the case of LED-B, an ideality factor of 2.3 was extracted. It is assumed that a leakage is occurred in the boundary interfaces between active layer and p-ZnMgSSe cladding layer or n-ZnMgSSe cladding layer. The evaluation of ideality factors is shown in Figure 6.

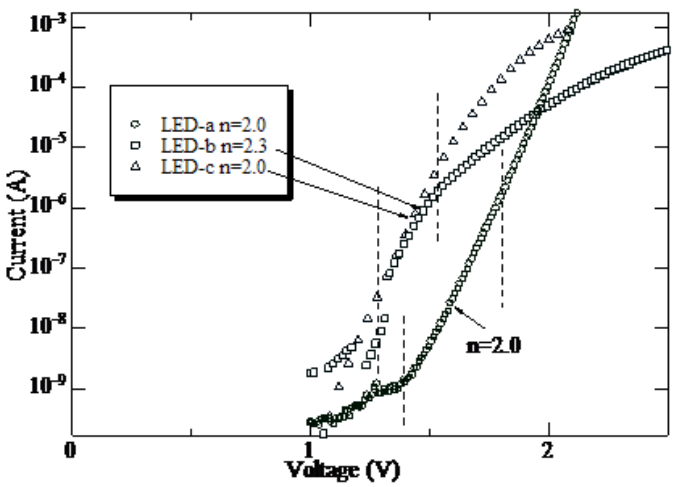

Figure 6: Ideality factor of ZnSSe:Te-based LEDs.

For evaluating the depletion width and effective carrier concentration, capacitancevoltage $(C-V)$ profiling was traced. Typical data for $C-V$ characteristics from the LED-A is shown in Figure 7. Using the general $C-V$ relation, the effective carrier concentration can be given by Eq. (3):

$$
\frac{1}{C^{2}}=\frac{2}{e \epsilon_{s} \epsilon_{o} A^{2}\left(N_{A}-N_{D}\right)}\left(V_{d}-V\right)
$$

where $C$ measured capacitance, $\epsilon_{s}$ is permittivity of ZnSe, $A$ is LED area. To determine the effective carrier concentration $\left(N_{A}-N_{D}\right)$, the $1 / C^{2}$ is plotted as a function of biased voltage where the slopes in this plot give $N_{A}-N_{D}$. So, using Eq. (3), the effective carrier concentration in the p-type ZnMgSSe layer was evaluated as $\sim 2.8 \times 10^{16} \mathrm{~cm}^{-3}$ for the present LED.

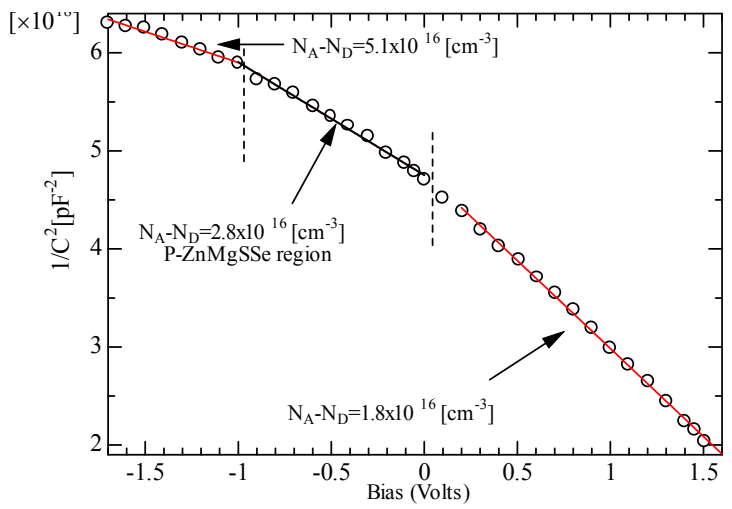

Figure 7: Typical C-V profile from ZnSSe:Te-based LED-A.

\section{Conclusions}

Blue-green and green ZnSSe:Te/Zn MgSSe DH-structure LEDs have been successfully fabricated grown by MBE, which has introduced the $\mathrm{ZnS}_{y} \mathrm{Se}_{1-\mathrm{x}-\mathrm{y}}$ : $\mathrm{Te}_{\mathrm{x}}$ $(x=0.04, y \sim 0.11-0.14)$ ternary epilayer as an active layer. Electroluminescent and electrical properties of the LEDs were systematically investigated. The electrolumine-scence(EL) characteristics for ZnSSe:Te-based LEDs at room temperature show blue-green (500 nm) and green $(\sim 525 \mathrm{~nm})$ light emission. From the 
$I-V$ characteristics, the built-in voltage $(\sim 2.1 \mathrm{~V})$ is very small compared to other wide bandgap LEDs, such as commercial InGaN-based LEDs $(>3.2 \mathrm{~V})$. From the $C-V$ profiling, the effective carrier concentration in the p-type ZnMgSSe cladding layer was evaluated as $\sim 2.8 \times 10^{16}$ $\mathrm{cm}^{-3}$ for the present LEDs.

Further works are required to establish optimum design of ZnSSe:Te active layer, including a band-offset between active and cladding layers.

\section{References}

[1] D.B. Eason, Z. Yu, W.C. Hughes, and W.H. Roland, "Molecular-beam epitaxy of high-quality ZnSe homo-epitaxial layers", Appl. Phys. Lett., 66, p.115. 1995.

[2] Z. Yu, D.B. Eason, C. Boney, and J. Ren, "High-brightness II-VI lightemitting diodes grown by molecular beam epitaxy", J. Vac. Sci. Technol., B 13, p.711, 1995.

[3] T. Yao. M. Kato, J.J. Davies, and H. Tanino, "Photoluminescence of Exciton Bound t Te Isoelectronic Traps in ZnSe", J. Cryst. Growth, 86, p.552, 1988.

[4] C.D. Lee, H.L. Park, C.H. Chung, and S.K. Chang, "Free-exciton Luminescence from ZnSe Zn-x $_{x} \mathrm{Te}^{\prime \prime}$, Phys. Rev. B, 45 p.4491, 1992.

[5] C.S. Yang, D.Y. Hong, C.Y. Lin, W.C. Chou, C.S. Ro, and S.L. Tu, "Optical Properties of the $\mathrm{ZnSe}_{1-x} \mathrm{Te}_{x}$ Epilayers Grown by Molecular Beam Epitaxy", J. Appl. Phys., 83, p.2555, 1988.

[6] H.C. Lee, T. Abe, N. Kaneko, M. Adachi, M. Watanabe, H. Kasada and K. Ando,"Efficient and Long-lived
Green Light-Emitting Diodes Based on ZnSSe:Te Active Layer", Japanese Journal of Applied Physics, vol. 41, p.1359, 2002.

[7] T. Abe, H. Ishikura, Y. Saomoto, K. Goto, K. Masuda, T. Shirai, H. Yamada, S. Kuroda, H. Kasada and K. Ando, "Optimization of ZnSe/ZnTe Superlattice Structured p-contact for ZnSe-based Optical Devices", Proceed. of 9th Inter. Conf. on II-VI compounds, Tu-p.053, 1999

[8] S. Nakamura, S. Pearto, and G. Fasol, "The blue laser diodes" Springer, p.97, 2000 .

\section{Author Profile}

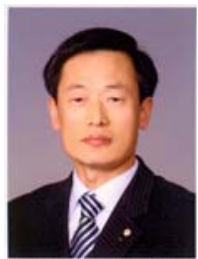

\section{Hong-Chan Lee}

$\mathrm{He}$ received his B. E. and M. Eng, degrees from Korea Maritime University, and his M. Eng. and Dr. Eng. from Tottroi University, Japan. He is currently an assistant professor in the faculty of energy \& resources engineering at Jungwon University. 